\title{
Analysis of Fretting Fatigue Crack Initiation in a Riveted Two Aluminum Specimen*
}

\author{
Li Huan ${ }^{1,2}$, Guo Ran ${ }^{2 \#}$, Cheng He Ming ${ }^{2}$, Wei Yeqing ${ }^{3}$ \\ ${ }^{1}$ College of Polytechnics, Yunnan Agriculture University, Kunming, China \\ ${ }^{2}$ Department of Engineering Mechanics, Kunming University of Science and Technology, Kunming, China \\ ${ }^{3}$ Yunnan Traffic Co., Ltd., Kunming, China \\ Email: 44997946@qq.com, "guoran99@mails.tsinghua.edu.cn, 86953734@qq.com
}

Received October 2013

\begin{abstract}
Based on the existing experiment results, the fretting fatigue contact geometry of a riveted two aluminum specimen was studied using the finite element method. The contact stress fields of the inner and outer contact edges on the two specimen's up and down surface under different contact friction coefficient and the fatigue loads were analyzed, the influences of the contact friction coefficient and remote stress on crack initiation were discussed. The results were in well agreement with the observations of the existing experiments, that is, the crack initiated places of the first aluminum specimen change from the area of 900 to 450 , and the crack initiated places of the second aluminum specimen change from the area of 900 to 1350 with the increase of the friction coefficient and the remote stress.
\end{abstract}

Keywords: A Riveted Two Aluminum Specimen; Crack Initiation; Frictional Coefficient; Fatigue Loads

\section{Introduction}

Fretting fatigue is one of the main reasons for the failure of structure components, even the causes of many major accidents in the fields of aviation, transportation and mechanics etc. The fretting fatigue begins with the wear and sometimes corrosion damage at the asperities of the contact surfaces of the riveted components, which will further induce the initiation and propagation of microcracks. With the appearance of the micro-cracks, the fatigue strength of the riveted components will significantly reduce, leading to the decrease of the components' service life [1]. Actually, the fretting fatigue is a damage process which is induced by the cyclic stress that works on the asperities of the materials' near-surface and causes the locally permanent structure deformations on the surface [2]. Under the action of the cyclic stress, the components immediately enter into a fatigue development process. The results of the damage accumulation during this process are the initiation and propagation of the cracks, following with the final fracture to end the process. The researches of fretting fatigue are very significant to ease fretting damage industry today. Due to the complicate deformation process and the difficulties on current experimental measurements, the researches of

*Project supported by the National Natural Science Foundation of China (Grant No. 11072092 and 11262007).

${ }^{*}$ Corresponding author. the stress states in the contact area are mainly resorted to the numerical simulation methods. For example, M.A.M cCarthy and C.T.M cCarthy etc [3] have constructed the FEM model of a 3-D bolt-alaminated plate to analyze the plate's contact stress. Mutoh, ect [4] analyzed the stress distribution in the contact areas of a specimen contacting with a rectangular fretting pad, based on which the fretting fatigue life of the specimen was studied by using fracture mechanics theories and was found to be well consistent with the experimental results.

Thus, following the methods and theories employed by Mutoh [4], a riveted two aluminum plate specimen was constructed and studied. The distributions of the normal stress and shear stress in the contact area between the two contacting aluminum plates were analyzed to find out the positions of the maximum stress and displacement.

\section{The Analysis of Influence Factors on Fretting Fatigue}

There are many factors influencing fretting fatigue, including all kinds of fretting parameters, displacement amplitude and environmental factors etc [5-8], the driving force of crack initiation is micro power, including tangential force caused by surface friction, the tensile stress and shear stress in the component body, and tensile stress and shear stress generated by contact pressure (namely the clamping force). The quantitative formula of 
driving force of crack growth [9] is:

$$
\sigma_{\mathrm{ff}}=\sigma_{\mathrm{f}-2} \mu \mathrm{P} 0(1-\mathrm{e}-\mathrm{S} / \mathrm{K})
$$

Type: $\mu$ is the contact surface friction coefficient, $\mathrm{P} 0$ is the contact pressure $(\mathrm{Pa}), \mathrm{S}$ is the fretting slip amplitude (M), $\mathrm{K}$ is formula constant having a length dimension (m). Specimens of stress which fatigue crack initiation required is $\sigma_{\mathrm{f}}$, load stress which lead to specimen's fatigue crack is $\sigma_{\mathrm{ff}} . \sigma_{\mathrm{ff}}$ is the component's fatigue strength fretting and $\sigma_{\mathrm{f}}$ is the component's fatigue strength no fretting. Fatigue strength in a micro component is smaller than which without fretting, which can be seen from this formula, and the difference between the two depends on $\mathrm{P} 0, \mu$ and $\mathrm{S}$, that is to say the fretting fatigue strength is related to friction coefficient, contact pressure and slip amplitude and so on, yet the slip amplitude is directly related to fatigue loading. So, based on the above mentioned theory, the fretting fatigue contact geometry of a riveted two aluminum specimen was studied using the finite element method. The contact stress fields of the inner and outer contact edges on the two specimen's up and down surface under different contact friction coefficient and the fatigue loads were analyzed, the influences of the contact friction coefficient and remote stress on crack initiation and propagation mechanism were discussed.

\section{Modeling}

\subsection{Computational Model}

The 3D finite element model of the rived aluminum specimen and its meshing result are showed in Figure 1. In order to reduce the computational cost, only half of the FEM model is constructed according to the symmetries of the specimen. The model is composed of 8 parts, including two aluminum plates, one screw bolt, one protective sleeve, two screw caps and two gaskets. In order to further reduce the model size and computational cost, the reducing of the meshing numbers and contact areas is $\rightarrow$ are often adopted in the FEM simulation. Thus, we treat the screw bolt, the protective sleeve, the screw caps and the gaskets as an integrated section to neglect the contacts between those parts. Three contacts regions are investigated in the simulations as shown in Figure 2: The first region is the contact area between the upper protective sleeve's lower surface and the aluminum plate I's upper surface; the second region is the contact area between the two aluminum plates; the third region is the area between the lower protective sleeve's upper surface and the aluminum plate II's lower surface. Among these three regions, the stress distributions of upper and lower surfaces of the two aluminum plates are emphatically analyzed to evaluate the specimen's fatigue life. The eight-node hexahedral solid elements are employed in the

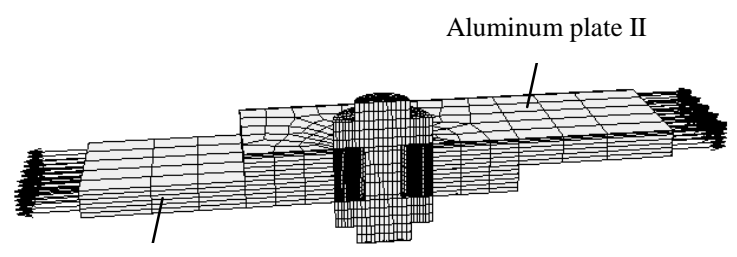

Aluminum plate I

Figure 1. FEM model of the rived aluminum specimen.

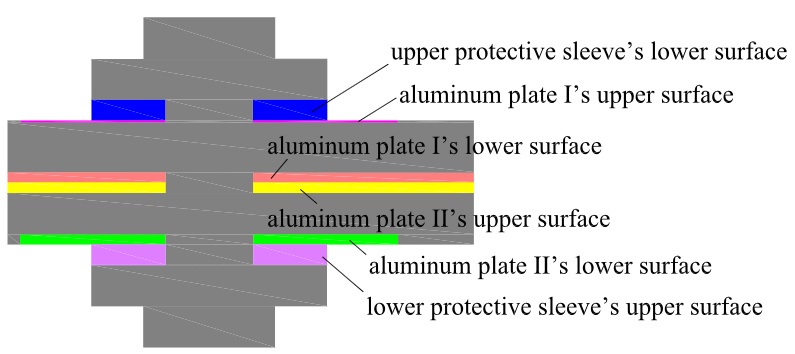

Figure 2. Contact regions.

simulation, where the total element number and node number are 10336 and 13686. The contact area of the two aluminum plates is $219.142 \mathrm{~mm}^{2}$ with a contact width of $4.5 \mathrm{~mm}$. The hole radius and thickness are set as $5.5 \mathrm{~mm}$ and $6 \mathrm{~mm}$, respectively. The length and width are respective $230 \mathrm{~mm}$ and $60 \mathrm{~mm}$. The aluminum plates' longitudinal axis is axis $\mathrm{X}$, forward direction points to longitudinal remote end. Transverse axis is axis $\mathrm{Y}$, the screw bolt's axis is axis Z, origin of coordinates is located in the centre of the hole. Normal chain bar constraints are exerted as boundary condition in fornter of the model $(\mathrm{y}=0)$.

\subsection{Mechanics of Material Constants}

In computing object this time, expect the two aluminum plates whose Young's modulus $E$ and Poisson's ratio $\mu$ are $40 \mathrm{GPa}$ and 0.3 , all the other parts of the specimen are C45 steels with $E=210 \mathrm{GPa}$ and $\mu=0.3$. Due to the elastic stress states of the screw bolt during its service process, the screw bolt is regarded as an elastic material in current simulation while the plasticity is taken into consideration for all the other parts of the specimen. Because the analytical objects are connected components in the fields of aviation and high speed train systems etc, so the work temperature is really the same as environmental temperature. Material temperature effect is not taken into consideration. Material constants of every component are showed in Table 1.

\subsection{Computational Method}

In order to simulate the fastening process of the screw bolt, the FEM model with a clearance of $d$ between the spaces of the two gaskets and the aluminum plate's 
Table 1. The mechanical parameters of the parts.

\begin{tabular}{cccc}
\hline parts & $\begin{array}{c}\text { protective sleeve, screw } \\
\text { caps and gaskets }\end{array}$ & screw bolt & specimen \\
\hline material & $\begin{array}{c}\text { C45 steels } \\
\text { (elastoplasticity) }\end{array}$ & $\begin{array}{c}\text { C45 steels } \\
\text { (elasticity) }\end{array}$ & aluminum \\
Young's modulus E & $210 \mathrm{GPa}$ & $210 \mathrm{GPa}$ & $70 \mathrm{GPa}$ \\
Poisson's ratio $\mu$ & 0.3 & 0.3 & 0.3 \\
\hline
\end{tabular}

thickness is constructed firstly. Through the adjustment of $\mathrm{d}$, different fastening forces could be simulated. The fastening process is realized through the following two steps: on the first step, the tension load is applied on the screw bolt to make the gaskets and the plates separate; on the second step, the tension load is released gradually to make the separation recover. During this recovery process, the gaskets would gradually fasten the aluminum plates when the contact relations between them are taken into consideration. The fatigue load is applied on the right end of the aluminum plate with the maximum stress of $\mathrm{s}=200 \mathrm{MPa}$ and fatigue load stress ratio of $\mathrm{R}=0.1$.

The contact stresses are studied using the direct constraint method, which would accurately track the movement of the contact bodies and the occurrence of the contact [10]. Once the contact occurs, the direct constraint method would directly adjust the displacements of the contact nodes by modifying their boundary conditions.

\section{Results and Discussion}

The contact surface is in compressive stress state when the shear stress of nodes in contact areas is negative value, which makes against fatigue crack initiation and propagation. The contact surface is in tensile stress state when the shear stress of nodes in contact areas is positive value, which provides the advantageous condition for fatigue crack initiation and propagation. In consideration of the larger relative slip distance of corresponding nodes in contact edges, The variational condition of the circumferential normal stress fields of the inner contact edges on the two specimen's up and down surface (this region are mainly subject to ordinary fatigue) and the shear stress fields of the outer contact edges on the two specimen's up and down surface (fretting fatigue region) are analyzed. Contact area is showed in Figure 3. The distributions of stress amplitude in the inner and outer circle contact edges of the aluminum plates under different friction coefficient and fatigue load conditions are discussed as follow.

\subsection{Influence of Friction Coefficient on the Initiation of Cracks}

To investigate the influence of friction coefficient on the

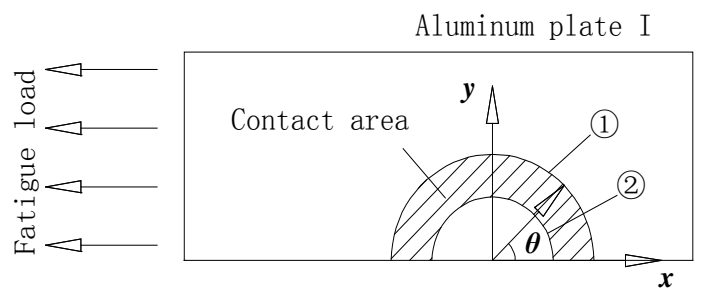

(a)

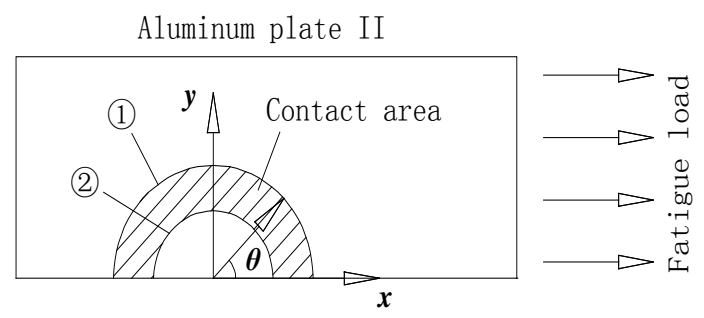

(b)

Figure 3. Contact area. (1) The outer circle contact edges; (2) The inner circle contact edges.

initiation of cracks in the contact region, the fastening force and fatigue stress are set constant as $5.5 \mathrm{KN}$ and $200 \mathrm{MPa}$, while three different friction coefficients, 0.35 , 0.5 and 0.7 , are considered. The maximum and minimum of the normal stress and shear stress on the aluminum plate I's lower surface and aluminum plate II's upper surface during one cycle of fatigue loading are analyzed to obtain the stress amplitudes $\left(\sigma_{\max }-\sigma_{\min }\right.$ and $\tau_{\max }-$ $\tau_{\min }$ ). The distributions of the stress amplitudes along the inner and outer circle contact edges of the two plates contact areas are calculated and shown in Figure 4. Based on Figure 4, the influence of friction coefficient on the stress amplitude can be obtained by analyzing the extreme values in those curves in Figure 4.

It can be seen from Figures 4(b) and (d) that the maximum normal stress amplitudes $\Delta \sigma_{\max }$ of the inner contact edges of the two aluminum plates locate at the about $90^{\circ}$ from the fatigue loading direction. However, it is interesting to find that the positions of $\Delta \sigma_{\max }$ on the plate I tend to change slightly toward the fatigue loading direction, while those on the plate II are just opposite, which tend to change slightly away from the fatigue loading direction. For both two plates, $\Delta \sigma_{\max }$ decrease with the increasing of friction coefficient. It indicates that the increasing of friction coefficient, on the one hand, would weaken the role of traditional fatigue damage on the aluminum plates and delay the corresponding crack initiation at the $90^{\circ}$ region near the hole; and on the other hand, would strengthen the role of fretting fatigue damage and lead to the transition of the crack initiation positions from $90^{\circ}$ point at the inner edge to $45^{\circ}$ point at the outer edge for plate I and from $90^{\circ}$ point at the inner edge to $135^{\circ}$ point at the outer edge for plate II. 


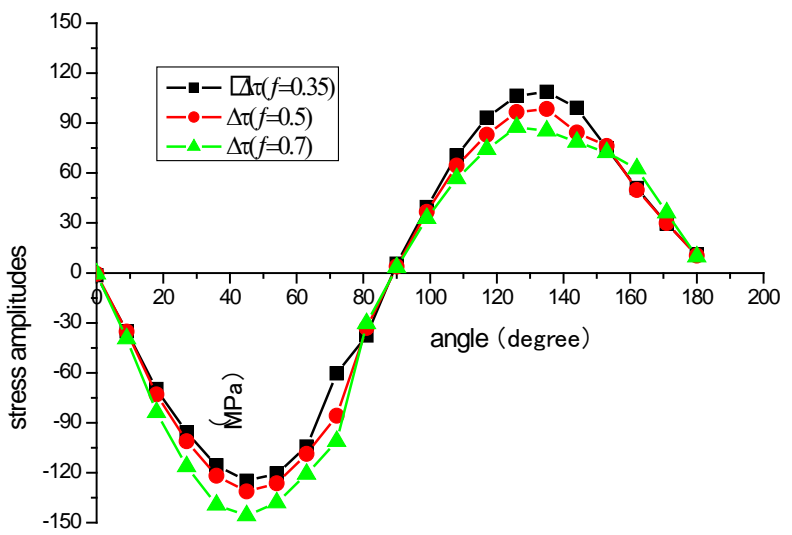

(a)

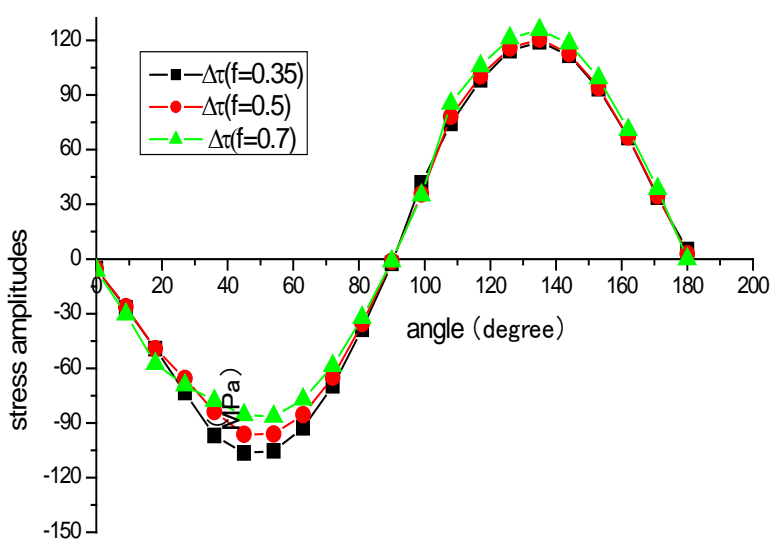

(c)

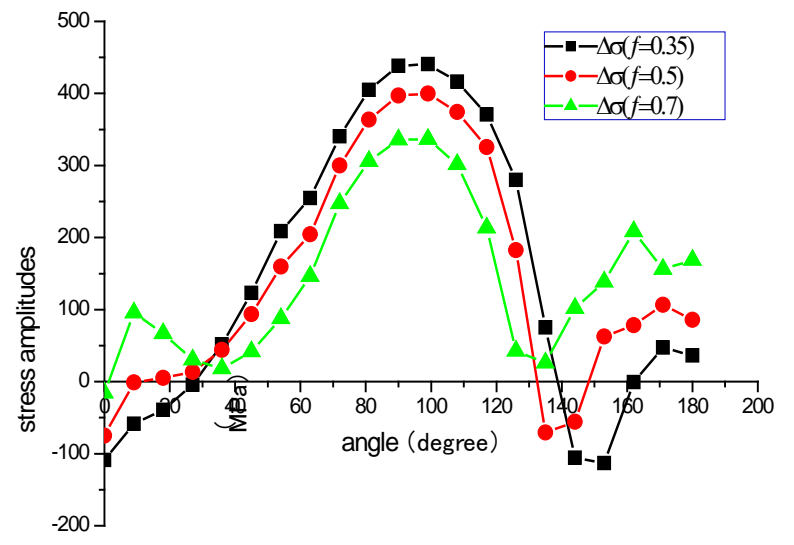

(b)

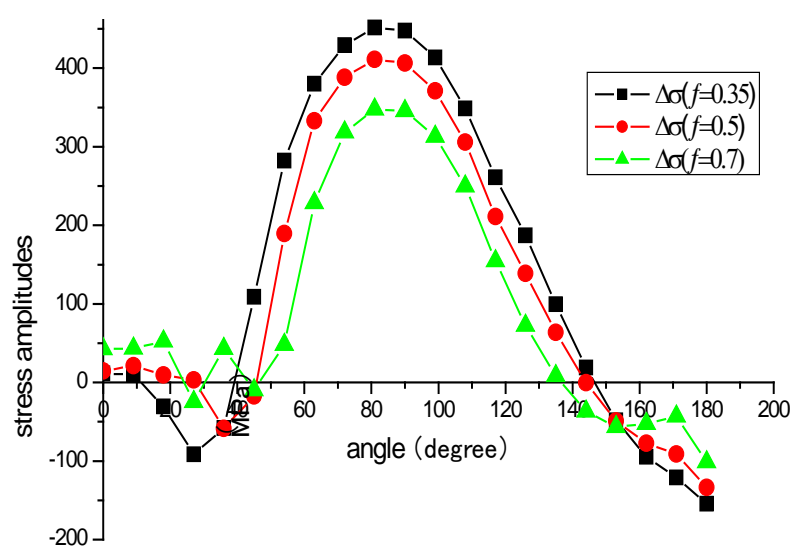

(d)

Figure 4. The distributions of stress amplitude in the inner and outer circle contact edges of the aluminum plates under different friction coefficient conditions. (a) The outer circle contact edges of the aluminum plate I's upper surface; (b) The inner circle contact edges of the aluminum plate I's upper surface; (c) The outer circle contact edges of the aluminum plate II's lower surface; (d) The inner circle contact edges of the aluminum plate II's lower surface.

It can be observed from Figures 4(a) and (c) that he maximum shear stress amplitudes $\Delta \tau_{\max }$ of the outer contact edges of plate I increase with the friction coefficient at $0^{\circ}-90^{\circ}$ region around the hole while decrease with the friction coefficient at $90^{\circ}-180^{\circ}$ region around the hole. Thus, when the fastening force and fatigue stress are fixed, increasing of the friction coefficient would increase the friction force at $45^{\circ}$ region of the plate I, improve the driving force to initiate and propagate the cracks and strengthen the role of fretting fatigue damage at this region. The situation is just opposite for plate II, $\Delta \tau_{\max }$ decrease with the friction coefficient at $0^{\circ}-90^{\circ}$ region while increase with the friction coefficient at $90^{\circ}$ $1800^{\circ}$ region. Thus, when the fastening force and fatigue stress are fixed, increasing of the friction coefficient would strengthen the role of fretting fatigue damage at $135^{\circ}$ region. From the above discussion, we can reach the conclusion that increasing the friction coefficient would result in the initiation of cracks at $45^{\circ}$ region of plate I's outer contact edge and the initiation of cracks at $135^{\circ}$ region of plate II's outer contact edge.

\subsection{Influence of Fatigue Load on the Initiation of Cracks}

To investigate the influence of fatigue load on the initiation of cracks in the contact region, the fastening force and friction coefficient are set constant as $4 \mathrm{KN}$ and 0.5 , while three different fatigue stresses, $150 \mathrm{MPa}, 200 \mathrm{MPa}$ and $250 \mathrm{MPa}$, are considered. Following the same analysis process in Section 4.1, the distributions of the stress amplitudes and along the inner and outer circle contact edges of the two plates contact areas are calculated and shown in Figure 5.

From Figures 5(b) and (d), it is easy to find that the maximum normal stress amplitudes $\Delta \sigma_{\max }$ of the inner contact edges of the two aluminum plates locate at about $90^{\circ}$ region near the hole, and the position of $\Delta \sigma_{\max }$ gradually shifts to the region whose angle is less than $90^{\circ}$ and more than $90^{\circ}$ as fatigue stress increases, which demon- 


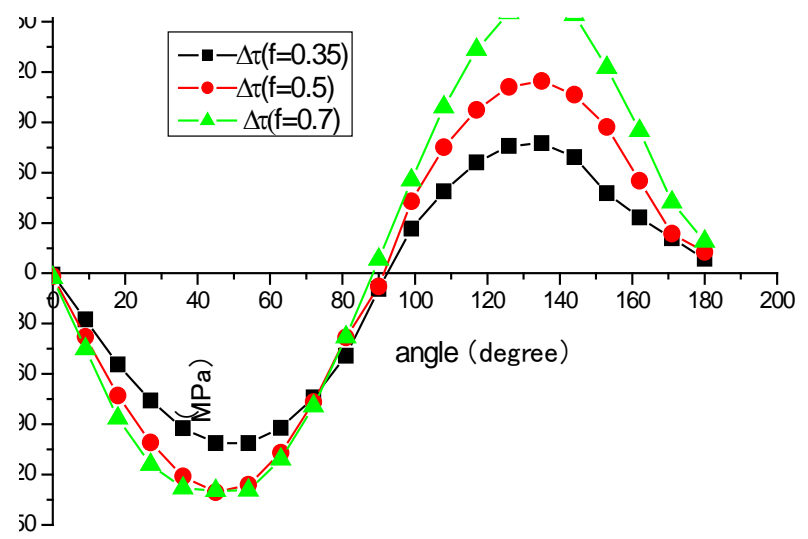

(a)

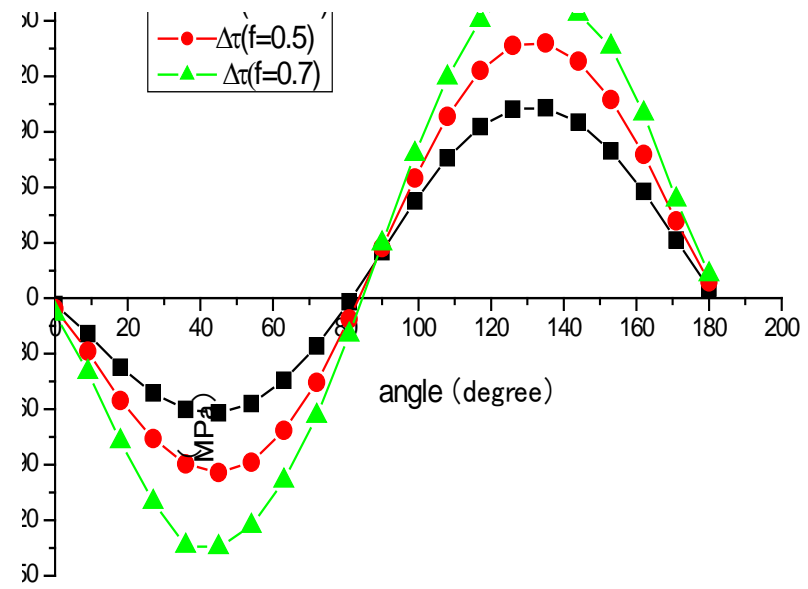

(c)

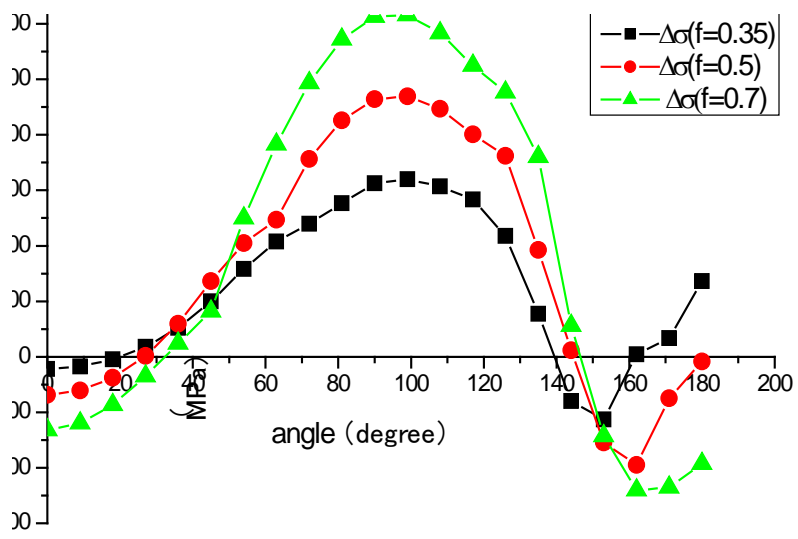

(b)

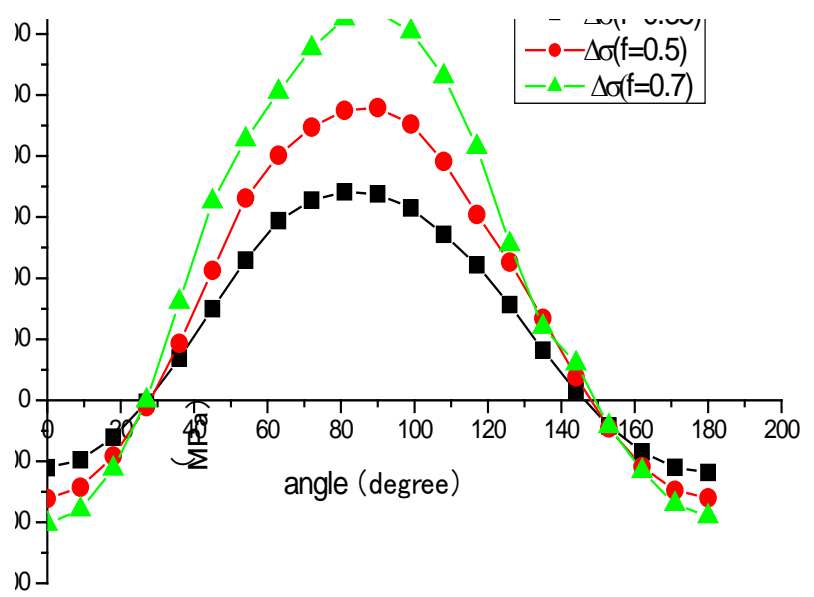

(d)

Figure 5. The distributions of stress amplitude in the inner and outer circle contact edges of the aluminum plates under different fatigue stress conditions; (a) The outer circle contact edges of the aluminum plate I's upper surface; (b) The inner circle contact edges of the aluminum plate I's upper surface; (c) The outer circle contact edges of the aluminum plate II's upper surface; (d) The inner circle contact edges of the aluminum plate II's upper surface.

strates that when the traditional fatigue plays major role for the failure of the specimen, increasing of fatigue stress would accelerate the initiation of the corresponding fatigue cracks at the inner contact edge and shift the initiation position far away from its original $90^{\circ}$ region.

For the maximum shear stress amplitude $\Delta \tau_{\max }$, we can observe from Figures 5(a) and (c) that $\Delta \tau_{\max }$ appear at about $45^{\circ}$ region and $135^{\circ}$ region, respectively and they both significantly increase with the fatigue stress. Meanwhile, the position of $\Delta \tau_{\max }$ can be seen to shift to the region whose angle is less than $45^{\circ}$ and the region whose angle is greater than $135^{\circ}$ with the fatigue stress. It indicates that increasing of fatigue stress would strengthen the role of the fretting fatigue damage at the outer contact edges, causing the cracks tend to initiate at $45^{\circ}$ region and $135^{\circ}$ region at outer contact edges and increase the tends to shift the crack initiation positions toward the region whose angle is less than $45^{\circ}$ or larger than $135^{\circ}$.

\section{Discussions}

The fretting fatigue contact geometry of a riveted two aluminum specimen was studied. The distributions of normal stress amplitude and shear stress amplitude in the inner and outer circle contact edges of the aluminum plates under different friction coefficient and fatigue load conditions are elastically analyzed using the finite element method and the following conclusions are obtained.

1) In the light of traditional fatigue damage without fretting damage, the main factor influencing fatigue is hoop normal stress amplitude $\Delta \sigma_{\max }$, the dangerous point of traditional crack initiation is just the place where hoop normal stress amplitude $\Delta \sigma_{\max }$ reached the maximum.

2) The aluminum plate I's fatigue crack initiation angle shifts from 900 to 450 with the increasing of friction coefficient and fatigue load, the aluminum plate II's fatigue crack initiation angle shifts from 900 to 1350 with the increasing of friction coefficient and fatigue load. The increasing of friction coefficient and fatigue load, on 
the one hand, would weaken the role of traditional fatigue damage on the aluminum plates and delay the corresponding crack initiation at the $90^{\circ}$ region near the hole; and on the other hand, would strengthen the role of fretting fatigue damage and lead to the transition of the crack initiation positions from $90^{\circ}$ point at the inner edge to $45^{\circ}$ point at the outer edge for plate I and from $90^{\circ}$ point at the inner edge to $135^{\circ}$ point at the outer edge for plate II.

3) Either traditional fatigue or fretting fatigue has been accelerated with the increasing of fatigue loads, the increasing of fatigue stress would increase the tends to shift the initiation position far away from its original $90^{\circ}$ region at the inner contact edge and shift the crack initiation positions toward the region whose angle is less than $45^{\circ}$ or larger than $135^{\circ}$ at the outer contact edge.

\section{REFERENCES}

[1] H. Zhao and Z. R. Zhou, "Application and Progress of Numerical Methods in Analysis of Fretting Fatigue," Tribology, Vol. 20, No. 4, 2000, pp. 317-320. (in Chinese)

[2] J. Liu, D. X. Liu and Y. Y. Liu, "Finite Element Analysis for Fretting Contacting Stresses,” Joumal of Mechanical Strength, Vol. 27, No. 4, 2005, pp. 504-509.

[3] M. A. McCarthy, C. T. McCarthy, V. P. Lawlor and W. F. Stanley, "Three-Dimensional Finite Element Analysis of Single-Bolt, Single-Lap Composite Bolted Joints: Part 1:
Model Development and Validation,” Composite Structure, Vol. 71, 2005, pp. 140-158. http://dx.doi.org/10.1016/j.compstruct.2004.09.024

[4] Y. Mutoh and J. Q. Xu, "Fracture Mechanics Approach to Fretting Fatigue and Problems to Be Solved,” Tribology International, Vol. 36, 2003, pp. 99-107. http://dx.doi.org/10.1016/S0301-679X(02)00136-6

[5] M. X. Shen, J. F. Peng, J. F. Zheng, C. Song, J. L. Mo and M. H. Zhu, "Study and Development of Fretting Fatigue,” Materials Engineering, 2010, p. 12.

[6] M. S. Yang, Y. L. Chen and Y. Q. Bi, "Study of Fretting Fatigue Influenceing Factors Based on Predictable Model of Fatigue Life," Journal of Naval Aeronautical and Astronautical University, Vol. 27, No. 1, 2012.

[7] D. R. Swalla and R. W. Neu, "Influence of Coefficient of Friction on Fretting Fatigue Crack Nucleation Prediction,” Tribology International, Vol. 34, 2001, pp. 493-503. http://dx.doi.org/10.1016/S0301-679X(01)00048-2

[8] O. Jin and S. Mall, "Effects of Slip on Fretting Behavior: Experiments and Analyses,” Wear, Vol. 256, 2004, pp. 671-684. http://dx.doi.org/10.1016/S0043-1648(03)00510-6

[9] D. Ning, Y. B. He and W. D. Yao, "Study and Analysis on Fretting Wear and Fretting Fatigue for Irradiation Surveillance Capsules in NPP,” Nuclear Power Engineering and Technology, Vol. 3, 2007, pp. 6-11.

[10] H. H. Chen and P. Qi, "Patran/Marc Training Course and Instance,” Science Press, Beijing, 2004. 